\title{
The reno-protective effect of a phosphoinositide 3-kinase inhibitor wortmannin on streptozotocin-induced proteinuric renal disease rats
}

\author{
Sang-Hoon Kim, Young-Woo Jang, \\ Patrick Hwang, Hyun-Jung Kim, Gi-Yeon Han \\ and Chan-Wha Kim ${ }^{1}$
}

\author{
School of Life Sciences and Biotechnology \\ Korea University \\ Seoul 136-701, Korea \\ ${ }^{1}$ Corresponding author: Tel, 82-2-3290-3439; \\ Fax, 82-2-3290-3957; E-mail, cwkim @korea.ac.kr \\ http://dx.doi.org/10.3858/emm.2012.44.1.004
}

Accepted 2 November 2011

Available Online 3 November 2011

\begin{abstract}
Abbreviations: ACR, albumin to creatinine ratio; CD2AP, CD2 associated protein; DN, diabetic nephropathy; ECM, expanded extracellular matrix; ESRD, end-stage renal disease; GBM, glomerular basement membrane; GFR, glomerular filtration rate; PAS, periodic acid-schiff; PI3K, phosphoinositide 3-kinase; SD, slit diaphragm; STZ, streptozotocin; SPRD, STZ-induced proteinuric renal disease
\end{abstract}

\begin{abstract}
Diabetic nephropathy (DN) is a progressive kidney disease that is caused by injury to kidney glomeruli. Podocytes are glomerular epithelial cells and play critical roles in the glomerular filtration barrier. Recent studies have shown the importance of regulating the podocyte actin cytoskeleton in early DN. The phosphoinositide 3-kinase (PI3K) inhibitor, wortmannin, simultaneously regulates Rac1 and Cdc42, which destabilize the podocyte actin cytoskeleton during early DN. In this study, in order to evaluate the reno-protective effects of wortmannin in early DN by regulating Rac1 and Cdc42, streptozotocin (STZ)-induced proteinuric renal disease (SPRD) rats were treated with wortmannin. The albuminuria value of the SPRD group was 3.55 $\pm 0.56 \mathrm{mg} /$ day, whereas wortmannin group was 1.77 $\pm 0.48 \mathrm{mg} /$ day. Also, the albumin to creatinine ratio (ACR) value of the SPRD group was $53.08 \pm 10.82$ $\mathrm{mg} / \mathrm{g}$, whereas wortmannin group was $20.27 \pm 6.41$ $\mathrm{mg} / \mathrm{g}$. Changes in the expression level of nephrin, podocin and Rac1/Cdc42, which is related to actin cytoskeleton in podocytes, by wortmannin administration were confirmed by Western blotting. The ex-
\end{abstract}

pression levels of nephrin (79.66 \pm 0.02$)$, podocin $(87.81 \pm 0.03)$ and Rac1/Cdc42 (86.12 \pm 0.02$)$ in the wortmannin group were higher than the expression levels of nephrin $(55.32 \pm 0.03)$, podocin $(53.40 \pm$ 0.06) and Rac1/Cdc42 (54.05 \pm 0.04$)$ in the SPRD group. In addition, expression and localization of nephrin, podocin and desmin were confirmed by immunofluorescence. In summary, we found for the first time that wortmannin has a reno-protective effect on SPRD rats during the early $D N$. The beneficial effects of wortmannin in SPRD rats indicate that this compound could be used to delay the progression of the disease during the early DN stage.

Keywords: albuminuria; diabetic nephropathies; nephrin; podocin; podocytes; wortmannin

\section{Introduction}

Diabetic nephropathy (DN) is a major complication caused by the development of diabetes mellitus, which damages the kidneys (Guilbert, 2006). It has been defined as increased protein excretion in urine (Mundel and Reiser, 2010). Major components of the glomerulus in the kidney are capillary endothelial cells, GBM, podocytes, and mesangial cells. The glomerulus filters proteins, cells and soluble water from the blood as they pass through the membrane. Various mechnisms lead to the progression and development of DN. The glomerulus is injured by glomerular hypertrophy, which is the thickening of the GBM, and the formation of ECM during early DN. These effects lead to glomerular hyperfiltration, hypertension, microalbuminuria and foot process effacement (Jefferson et al., 2008).

Among the components of the glomerulus, podocytes are terminally differentiated and highly specialized cells in the Bowman's capsule in the kidney that wrap around the capillaries of the glomerulus (Pavenstädt et al., 2003). Podocytes form the glomerular filtration barrier through the formation of a foot process, which is permeable to molecules smaller than albumin (Tryggvason and Wartiovaara, 2001). Podocytes provide structural support to the glomerular tuft and maintain the permeability properties of the filtration barrier 
A

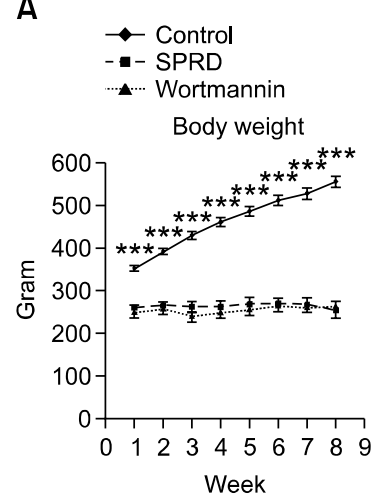

B

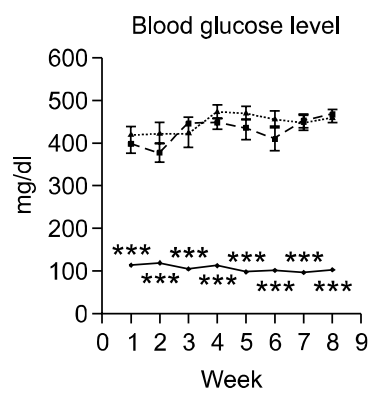

Figure 1. Body weight and blood glucose level in normal control, SPRD and wortmannin-treated rats. (A) Changes of body weight in rats over 8 weeks for each group. (B) Changes of blood glucose level in rats over 8 weeks for each group. Significant differences were determined by ANOVA (means \pm SEM, $n=10$ ) with Duncan post hoc multiple comparison test using the SPSS 12.0 for Windows computer software package (SPSS, Chicago, IL). ${ }^{* * *} P<0.001$; control vs. SPRD and wortmannin).

(Farquhar, 2006). Podocyte injury during the early DN stage leads to structural changes associated with actin cytoskeleton, such as foot process effacement and SD disruption (Smoyer and Mundel, 1998). The structural changes in the podocyte morphology during the early DN stage is completely reversible (Mundel and Reiser, 2010). Recent studies have shown that regulation of the kidney podocyte actin cytoskeleton is important during the early DN stage (Schlondorff, 2008).

$\mathrm{PI} \mathrm{K}$ is a family of enzymes involved in cellular functions such as cell growth, proliferation, differentiation, and motility (Yousif, 2008). PI3K is also a major component of the insulin signaling pathway. Treatment of diabetes mellitus through PI3K signaling pathway has recently been of great interest. $\mathrm{PI} 3 \mathrm{~K}$ simultaneously regulates Rac1 and Cdc42, which are associated with the actin cytoskeleton of kidney podocytes (Zhu et al., 2008). All PI3Ks are inhibited by the drugs wortmannin and LY294002. Wortmannin, a furanosteroid metabolite of the fungi Penicillium funiculosum, Talaromyces (Penicillium) wortmannii, is a specific, covalent inhibitor of PI3Ks. The half-life of wortmannin in tissue culture is about 10 minutes due to the presence of the highly reactive $\mathrm{C}_{20}$ carbon, which is responsible for its ability to covalently inactivate PI3K. Wortmannin is a commonly used cell biology reagent and has been previously used to inhibit DNA repair, receptor-mediated endocytosis and cell proliferation (Liu et al., 2005). Therefore, in this study we investigated the reno-protective effect of wortmannin on SPRD rats in vivo.

A

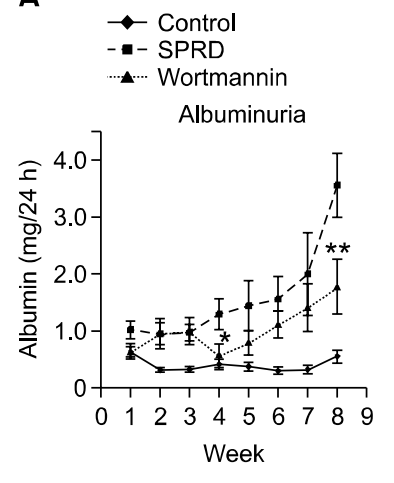

B

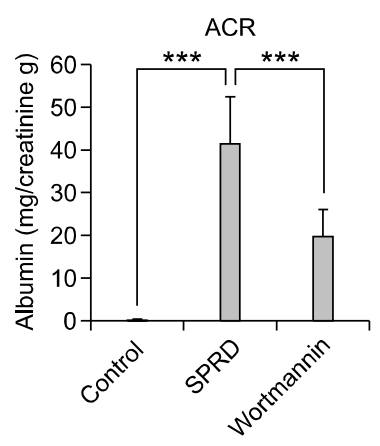

Figure 2. Albuminuria and albumin to creatinine ratio (ACR) in normal control, SPRD and wortmannin-treated rats. (A) Analysis of albuminuria changes in rats over 8 weeks for each group through ELISA method. (B) Analysis of ACR changes in rats after sacrifice for each group through ELISA method. Significant differences were assessed via ANOVA (means \pm SEM, $n=10$ ) with the SPSS 12.0 statistical software package (SPSS, Chicago, IL). $\left({ }^{*} P<0.05 ;{ }^{* *} P<0.01 ;{ }^{* * *} P<0.001\right)$.

\section{Results}

\section{Wortmannin decreases albuminuria and ACR}

After induction of type 1 diabetes, the body weight and blood glucose level of the rats were measured every week. Weight loss in the SPRD group and the wortmannin group were observed over the 8 weeks study period (Figure 1A). The blood glucose levels in the SPRD group (430-600 mg/dl) and wortmannin group $(460-600 \mathrm{mg} / \mathrm{dl})$ were significantly higher than those of the control group. (Figure 1B). The body weight and blood glucose level were not changed by wortmannin treatment.

After 8 weeks of treatment with wortmannin, the albuminuria value $(1.77 \pm 0.48 \mathrm{mg} / \mathrm{dl})$ in the wortmannin group was decreased when compared with the SPRD group $(3.55 \pm 0.56 \mathrm{mg} / \mathrm{dl})$ (Figure 2A). In addition, the ACR of the wortmannin group (20.27 \pm 6.41 albumin $\mathrm{mg} /$ creatinine g) was significantly lower than the SPRD group (53.08 \pm 10.82 albumin $\mathrm{mg} / \mathrm{creatinine} \mathrm{g}$ ) (Figure 2B). Based on the albuminuria and $A C R$ results, we demonstrated that wortmannin treatment can lower the albuminuria and ACR when compared with the SPRD group. Therefore, wortmannin may have a renoprotective effect by lowering albuminuria and ACR.

\section{Histopathological changes in glomerulus recovered by wortmannin treatment}

Periodic acid-Schiff (PAS) is a staining method used to detect glycogen in tissues. The PASstained kidney sections of rats were examined under a microscope. We detected histological 
evidence of more injury to the kidney glomeruli of the SPRD group than the control. Glomerular hypertrophy, which can be detected by minimal accumulation of mesangial matrix and GBM

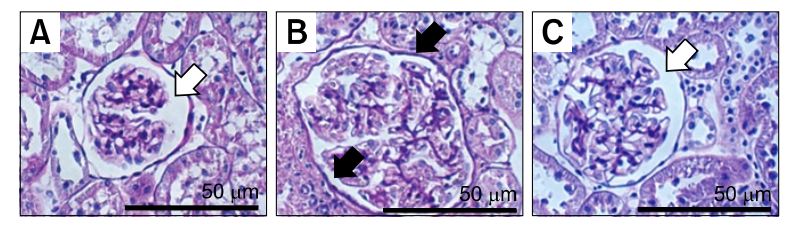

D

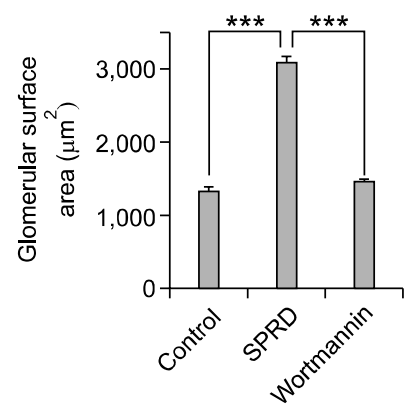

Figure 3. Histopathological changes of the glomeruli by wortmannin and glomerular surface area in normal control, SPRD and wortmannin-treated rats. (A) Control group, $\times 400$; (B) SPRD group, $\times 400$; (C) Wortmannin group, $\times 400$; (D) Wortmannin group, $\times 400$. Black arrows indicate that glomerular hypertrophy, mesangiial matrix expansion and GBM thickening of SPRD group. White arrows show the normal glomeruli of the control and wortmannin group without histopathological changes such as glomerular hypertrophy, mesangiial matrix expansion and GBM thickening. (D) The glomerular surface area was calculated by multiplying the maximum width of the glomerulus diameter by the maximum length of glomerulus. significant differences were assessed via ANOVA (means \pm SEM, $n=30$ ) with the SPSS 12.0 statistical software package (SPSS, Chicago, IL) $\left.{ }^{* * *} P<0.001\right)$. thickening, were observed in the kidney sections of the SPRD group (Figure 3B). PAS (positive glycogen) accumulation was observed in the mesangial matrix in the untreated SPRD group; however, they were markedly decreased in the wortmannin treated group (Figure 3C). Therefore, wortmannin treatment may have reversed the histopathologic changes that occurred in the kidney of the SPRD group, such as glomerular hypertrophy, minimal accumulation of mesangial matrix and GBM thickening.

The glomerular surface area was calculated based on the PAS staining images. The glomerular surface area was measured to evaluate glomerular hypertrophy (Figure 3D). The SPRD group showed major pathological alterations including widening of the mesangial regions, mesangial matrix expansion, and thickening of the GBM. Thus, the glomerular surface area of the SPRD group $(3,111 \pm 75$ $\mu \mathrm{m}^{2}$ ) also markedly increased due to pathological alterations. However, the pathological alterations described above were improved after administration of wortmannin. As mentioned earlier, the glomerular surface area of the wortmannin group $\left(1,479 \pm 22 \mu \mathrm{m}^{2}\right)$ was completely restored to the normal condition. These results indicate that wortmannin produces reno-protective effects.

\section{Actin-cytoskeleton related proteins are differently expressed by wortmannin treatment}

Expression of nephrin (Figure 4A), podocin (Figure 4B) and Rac1/Cdc42 (Figure 4C) were confirmed by Western blotting. Western blot analysis of each protein was conducted on 10 cortex samples at 8 weeks for the control group, 10 cortex samples at 8
A

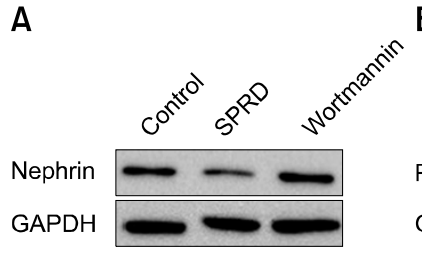

D

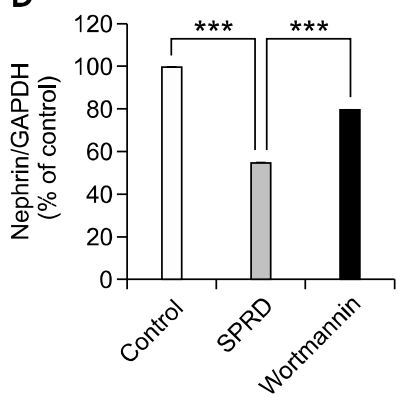

B

Podocin

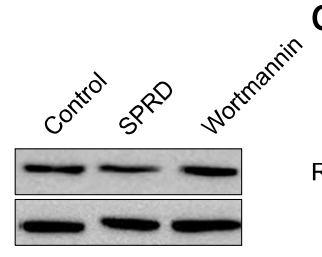

E

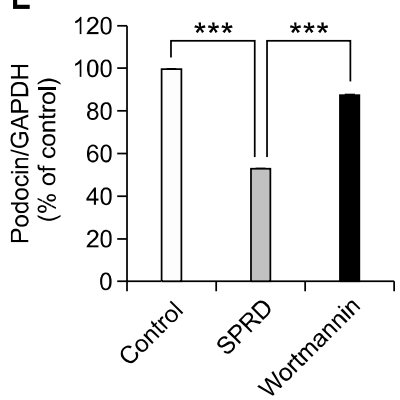

C

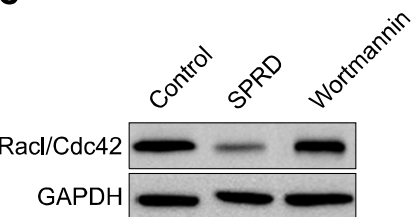

$\mathbf{F}$

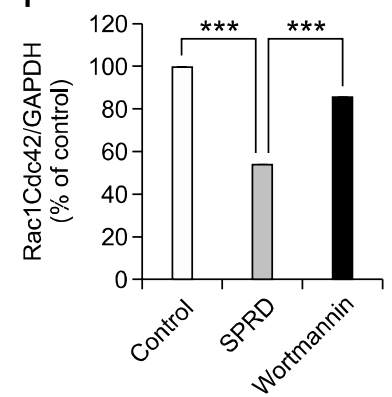

Figure 4. The effect of wortmannin on the expression of nephrin, podocin and Rac1/Cdc42 expression levels in SPRD rats via Western blotting. (A-C) Upper panel shows the nephrin, podocin, and Rac1/ Cdc42 band intensity of each group (control, SPRD, wortmannin). Lower panel shows the GAPDH band intensity of each group. (D-F) The band intensity was measured using the Multi Gauge V3.0 software (FUJIFILM). Significant differences were assessed via ANOVA (means \pm SEM, $n=3$ ) with the SPSS 12.0 statistical software package (SPSS, Chicago, IL) $\left({ }^{* * *} P<0.001\right)$. 

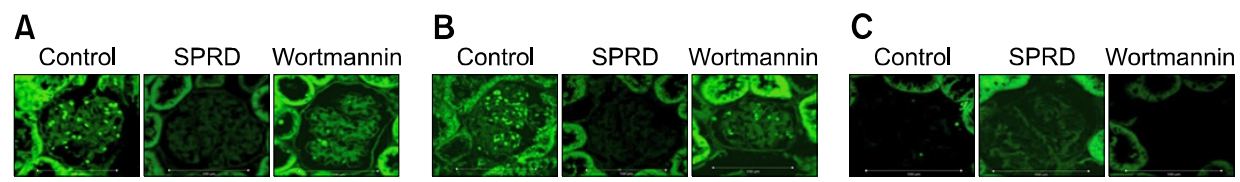

Figure 5. The effect of wortmannin on the expression and distribution of podocyte injury marker via immunofluorescence. (A) Nephrin, (B) Podocin, (C) Desmin.

weeks for the SPRD group and 10 cortex samples at 8 weeks for the wortmannin group. The expression of the slit diaphragm proteins, nephrin $(79.66$ $\pm 0.02)$ and podocin $(87.81 \pm 0.03)$ was significantly increased in the wortmannin group when compared with nephrin $(55.32 \pm 0.03)$ and podocin $(53.40 \pm 0.03)$ of the SPRD group (Figures 4A, 4B, $4 \mathrm{D}$ and $4 \mathrm{E})(P<0.001)$. The expression of GTPases, Rac1 and Cdc42 was significantly increased in the wortmannin group $(86.12 \pm 0.02)$ when compared with the SPRD group (54.05 \pm $0.04)$ (Figures $4 \mathrm{C}$ and $4 \mathrm{~F})(P<0.001)$.

\section{Wortmannin ameliorates the expression and localization of slit diaphragm proteins}

The localization and expression of nephrin, podocin and desmin in the kidney glomeruli were confirmed by immunofluorescence. The expression of nephrin and podocin were lower in the glomeruli of the SPRD group when compared to the control group and abnormal localization was also observed. In the wortmannin-treated group, the expression of nephrin and podocin were higher than the untreated SPRD group (Figures 5A and 5B). In addition, another podocyte injury marker, the expression of desmin, was increased in the glomeruli of the SPRD group when compared to the control group (Figure 5C). These results indicate that wortmannin ameliorates podocyte injury in SPRD rats via recovery of the expression and localization of podocyte injury marker proteins.

\section{Discussion}

The glomerular filtration barrier is a unique organization formed by a three-dimensional framework of podocytes (Simons et al., 2009). Podocytes, which are terminally differentiated cells that wrap around the glomerular capillaries, play a critical role in the glomerular filtration barrier. Structurally, podocytes form a cell body through primary and secondary foot processes. The foot processes interdigitate between adjacent processes and are connected by slit diaphragm proteins.

The molecular mechanisms of foot process effacement are not yet fully understood, although proposed mechanisms include disruption of the podocyte actin cytoskeleton (Kaplan et al., 2000), loss of slit diaphragm proteins (Saleem et al., 2002), or impediment of the podocyte-GBM interaction (Regele et al., 2000). However, it is still unclear if foot process effacement leads to proteinuria simply through podocyte injury. Proteinuria occurs when the permeability of the filtration barrier is increased in the glomerulus (Farquhar, 2006). Mutations affecting slit diaphragm proteins, including nephrin (Kestila et al., 1998), podocin (Boute et al., 2000), CD2AP (Löwik et al., 2007), TRPC6 (Reiser et al., 2005), PLCE1 (Hinkes et al., 2006) and $\alpha$-actinin-4 (Kaplan et al., 2000) lead to renal disease due to disruption of the filtration barrier and destabilization of the podocyte actin cytoskeleton (Faul et al., 2007). Of all the slit diaphragm proteins, nephrin is a critical component for maintaining the filtration barrier function (Kestila et al., 1998). Previous studies have reported a decrease in nephrin expression in other disease models (PAN), but not in the SPRD model (Kawachi et al., 2000, 2003). Also, reagents such as fluvastatin (Shibata et al., 2006), tolvaptan (Okada et al., 2009) and triptolide (Zheng et al., 2008) have been shown to have a reno-protective effect on actin cytoskeleton rearrangement by PAN. In this study, we administered STZ to SD-rats to induce DN. STZ has been used in medical research to produce an animal model of Type 1 diabetes. SPRD rats were confirmed based on blood glucose levels and albuminuria. In previous studies, the expression level of nephrin and podocin were significantly decreased in SPRD models (Cai et al., 2010; Chen et al., 2011). In addition, desmin was used as a marker of podocyte injury because of its reverse expression pattern with nephrin and podocin.

Here, we hypothesized that the PI3K inhibitor, wortmannin, may produce reno-protective effects by reducing albuminuria and restoring podocyte injury in SPRD rats. The results of this study demonstrates that wortmannin ameliorates albuminuria, ACR and histopathological changes such as mesangial matrix expansion, GBM thickening and glomerular hypertrophy. Wortmannin also re- 
covered expression of podocyte actin cytoskeleton related proteins in SPRD rats. The changes in protein expression levels in podocytes by treatment with wortmannin were confirmed via Western blotting and immunofluorescence. The loss of slit diaphragm proteins, nephrin and podocin, induced by STZ, were significantly increased by treatment with wortmannin. However, desmin was increased in SPRD rats, was remarkably reduced by treatment with wortmannin. In addition, down-stream proteins of PI3K and small GTPases, Rac1 and Cdc42 were restored in SPRD rats after treatment with wortmannin. These findings indicate that the reno-protective effects of wortmannin may be mediated through the Rac1 and Cdc42 pathway.

The PI3K inhibitor, wortmannin simultaneously regulate Rac1 and Cdc42 which is associated with the actin cytoskeleton of kidney podocytes. Rac1 is a small signaling $G$ protein, and is a member of the Rac subfamily of the Rho family of GTPases. Members of this superfamily appear to regulate a diverse array of cellular events, including the control of cell growth, cytoskeletal reorganization, and the activation of protein kinases. Cdc42 is also a GTPase of the Rho-subfamily, which regulates signaling pathways that control diverse cellular functions including cell morphology, migration, endocytosis and cell cycle progression. This protein could regulate actin polymerization by directly binding to N-WASP, which subsequently activates the Arp2/3 complex. The appropriate regulation of GTPase is very important for maintaining differentiation of podocytes (Gundersen et al., 1980). The other GTPases, RhoA, has already been extensively studied in this field (Schmieder et al., 2004; Asanuma et al., 2006; Shibata et al., 2006; Hidaka et al., 2008). Although the role of RhoA in diabetic renal diseases has been evaluated, changes in expression and activity of Rac1/Cdc42 in diabetic kidneys have not been reported. Based on the results of this study, adequate amounts of PI3K in healthy kidneys regulate the expression of Rac1/ Cdc42, whereas over expression is considered to inhibit their regulation. Therefore, we hypothesized that down-stream factors of $\mathrm{PI} 3 \mathrm{~K}$ and other GTPases; Rac1 and Cdc42, might also serve as regulation factors of podocyte actin cytoskeleton. We found that wortmannin treatment restored expression of Rac1 and Cdc42, which were lower in SPRD rats, via Western blotting. These findings suggest that restoration of the expression levels of Rac1 and Cdc42 mediate the reno-protective effects of wortmannin. Activation of Rac1 and Cdc42 may lead to destabilization of actin cytoskeleton in podocyte and albuminuria by disruption of the slit diaphragm. The reno-protective effects by wort- mannin was demonstrated by the recovery of expression and distribution of slit diaphragm proteins, nephrin, podocin, and the down-stream factors of PI3K, Rac1 and Cdc42 were also restored by treatment with wortmannin.

In conclusion, wortmannin ameliorated STZ-induced podocyte injury and microalbuminuria. Wortmannin was shown to improve diabetic nephropahy in the early stages through the Rac1 and Cdc42 pathway. Further studies are needed to determine the changes in Rac1/Cdc42 activity by PI3K inhibitor.

\section{Methods}

\section{Animals}

All animal procedures were performed according to the guidelines provided by the committee of animal ethics approved by the University of Korea. Thirty-male SD rats (6 weeks, $170-200 \mathrm{~g}$ ) were used in this experiment (Orient, Korea). The animals were fed a standard chow and water was freely available. They were maintained under the standard conditions of a 12-light-dark cycle, temperature at $23 \pm 1{ }^{\circ} \mathrm{C}$ and humidity at $50 \pm 10 \%$ (Choi et al., 2011). Rats were randomly divided into the following three groups; control, SPRD and wortmannin group $(n=10$ for each group). Type 1 diabetes mellitus was induced by an intraperitoneal injection of STZ (65 mg/kg; Sigma-Aldrich), which was dissolved in $\mathrm{pH} 4.5$ sodium citrate buffer. Control rats were injected with an equivalent volume of sodium citrate buffer only. Animals were considered diabetic and included for study only if plasma glucose levels were $\geq 300 \mathrm{mg} / \mathrm{dl} 3$ days following STZ injection (Lee et al., 2003). Wortmannin (1 mg/kg; LC laboratories) was administered to SPRD rats by intraperitoneal injection every day during 8 weeks. The wortmannin was dissolved in dimethyl sulfoxide (DMSO). Control and SPRD group rats were injected with an equivalent volume of DMSO only. The rats were sacrificed after 8 weeks.

\section{Albuminuria and albumin to creatinine ratio (ACR)}

Albuminuria was measured weekly over 8 weeks. To collect the urine samples, rats were placed in individual metabolic cages for $24 \mathrm{~h}$. After measurement of the volume, the urine samples were centrifuged at $4^{\circ} \mathrm{C}$ and $2,000 \mathrm{rpm}$ for $10 \mathrm{~min}$. The urinary albumin and creatinine concentrations in the supernatant was measured using an Enzyme-linked immunosorbent assay (ELISA) kit (Bethyl laboratories INC.) (Neuman and Cohen, 1989), respectively. ACR was calculated based on the albuminuria and urine creatinine level (albumin mg/creatinine g) (Kestenbaum and de Boer, 2010).

\section{Renal pathology}

The kidney tissues were fixed in $10 \%$ formalin, and embedded in paraffin. Kidney sections $4 \mu \mathrm{m}$ thick were made using a rotary microtome (Leitzer Wetzler) and stained with 
PAS for histological examination by light microscopy (OLYMPUS, CK2, Japan). This method was performed based on a modified method reported by Kim et al. (2007b) The glomerular surface area was measured to evaluate glomerular hypertrophy as described by Inada et al. (2005) For each group, 50 glomeruli per mid-transverse section of the kidney cortex were measured. The glomerular surface area was calculated by multiplying the maximum width of the glomerulus by the maximum length of the glomerulus.

\section{Western blotting analysis}

The protein was isolated from kidney cortex by sonication in RIPA buffer containing protease inhibitor cocktail solution. For immunoblotting, nephrin, podocin and Rac1/ Cdc42 were separated by SDS-PAGE, and then transferred to NC membranes. The membrane was incubated with blocking solution containing 1:2,000 dilution of anti-nephrin antibody (Abcam, Cambridge, UK), 1:500 dilution of anti-podocin antibody (Abcam), and 1:1,000 dilution of anti-Rac1/Cdc42 antibody (Cell signaling) and then incubated with blocking solution containing 1:2,000 (for nephrin and podocin), 1:1,000 (for Rac1/Cdc42) dilution of horseradish peroxidase-conjugated goat anti-rabbit IgG secondary antibody (Pierce Biotechnology Inc.), respectively. Rac1/Cdc42 antibody with sequence similarities that recognized endogenous levels of total Rac1 and Cdc42 proteins were used in Western blots. Gel bands from Western blot were scanned with LAS, and analyzed using Multi Gauge image analysis software (Fuji Film) (Cho et al., 2007; Kim et al., 2007a).

\section{Immunofluorescence}

Immunostaining for nephrin, podocin and desmin was performed using 1:100 dilution of rabbit polyclonal anti-rat nephrin antibody (Abcam), 1:100 dilution of rabbit polyclonal anti-rat podocin (Abcam) and 1:100 dilution of rabbit polyclonal anti-rat desmin (Abcam), respectively. The method used for immunostaining was previously described by Kim et al., with minor modifications (2007b).

\section{Statistical analysis}

All results are expressed as means \pm SEM. Comparison of continuous variables between more than two groups was performed using ANOVA, and a $t$-test was used to assess differences between groups. $P<0.05$ was considered statistically significant. The SPSS software package was used for the statistical test.

\section{Acknowledgements}

This study was supported by BK21 (Brain Korea 21). We thank center for analytical and lifescience instruments in the Korea University.

\section{References}

Asanuma K, Yanagida-Asanuma E, Faul C, Tomino Y, Kim
K, Mundel P. Synaptopodin orchestrates actin organization and cell motility via regulation of RhoA signalling. Nat Cell Biol 2006;8:485-91

Boute N, Gribouval O, Roselli S, Benessy F, Lee H, Fuchshuber A, Dahan K, Gubler MC, Niaudet P, Antignac C. NPHS2, encoding the glomerular protein podocin, is mutated in autosomal recessive steroid-resistant nephrotic syndrome. Nat Genet 2000;24:349-54

Cai Y, Chen J, Jiang J, Cao W, He L. Zhen-wu-tang, a blended traditional Chinese herbal medicine, ameliorates proteinuria and renal damage of streptozotocin-induced diabetic nephropathy in rats. J Ethnopharmacol 2010;131:88-94

Chen $\mathrm{KH}$, Hung CC, Hsu HH, Jing YH, Yang CW, Chen JK. Resveratrol ameliorates early diabetic nephropathy associated with suppression of augmented TGF-beta/smad and ERK $1 / 2$ signaling in streptozotocin-induced diabetic rats. Chem Biol Interact 2011;190:45-53

Cho EH, Kim MR, Kim HJ, Lee DY, Kim PK, Choi KM, Ryu $\mathrm{OH}$, Kim CW. The discovery of biomarkers for type 2 diabetic nephropathy by serum proteome analysis. Proteomics Clin Appl 2007;1:352-61

Choi R, Kim BH, Naowaboot J, Lee MY, Hyun MR, Cho EJ, Lee ES, Lee EY, Yang YC, Chung CH. Effects of ferulic acid on diabetic nephropathy in a rat model of type 2 diabetes. Exp Mol Med 2011;43:676-83

Farquhar MG. The glomerular basement membrane: not gone, just forgotten. J Clin Invest 2006;116:2090-3

Faul C, Asanuma K, Yanagida-Asanuma E, Kim K, Mundel $P$. Actin up: regulation of podocyte structure and function by components of the actin cytoskeleton. Trends Cell Biol 2007; 17:428-37

Guilbert JJ. The World Health Report 2006: working together for health. Educ Health (Abingdon) 2006;19:385-7

Gundersen HJ, Seefeldt T, Osterby R. Glomerular epithelial foot processes in normal man and rats. Distribution of true width and its intra- and inter-individual variation. Cell Tissue Res 1980;205:147-55

Hidaka T, Suzuki Y, Yamashita M, Shibata T, Tanaka Y, Horikoshi S, Tomino Y. Amelioration of crescentic glomerulonephritis by RhoA kinase inhibitor, Fasudil, through podocyte protection and prevention of leukocyte migration. Am J Pathol 2008;172:603-14

Hinkes B, Wiggins RC, Gbadegesin R, Vlangos CN, Seelow D, Nurnberg G, Garg P, Verma R, Chaib H, Hoskins BE, Ashraf S, Becker C, Hennies HC, Goyal M, Wharram BL, Schachter AD, Mudumana S, Drummond I, Kerjaschki D, Waldherr R, Dietrich A, Ozaltin F, Bakkaloglu A, Cleper R, Basel-Vanagaite L, Pohl M, Griebel M, Tsygin AN, Soylu A, Muller D, Sorli CS, Bunney TD, Katan M, Liu J, Attanasio M, O'Toole JF, Hasselbacher K, Mucha B, Otto EA, Airik R, Kispert A, Kelley GG, Smrcka AV, Gudermann T, Holzman LB, Nurnberg P, Hildebrandt F. Positional cloning uncovers mutations in PLCE1 responsible for a nephrotic syndrome variant that may be reversible. Nat Genet 2006;38:1397-405

Inada A, Nagai K, Arai H, Miyazaki J, Nomura K, Kanamori $H$, Toyokuni S, Yamada Y, Bonner-Weir S, Weir GC, Fukatsu A, Seino Y. Establishment of a diabetic mouse model with 
progressive diabetic nephropathy. Am J Pathol 2005;167: 327-36

Jefferson JA, Shankland SJ, Pichler RH. Proteinuria in diabetic kidney disease: a mechanistic viewpoint. Kidney Int 2008;74:22-36

Kaplan JM, Kim SH, North KN, Rennke H, Correia LA, Tong $\mathrm{HQ}$, Mathis BJ, Rodriguez-Perez JC, Allen PG, Beggs AH, Pollak MR. Mutations in ACTN4, encoding alpha-actinin-4, cause familial focal segmental glomerulosclerosis. Nat Genet 2000;24:251-6

Kawachi H, Koike H, Kurihara H, Sakai T, Shimizu F. Cloning of rat homologue of podocin: expression in proteinuric states and in developing glomeruli. J Am Soc Nephrol 2003;14: 46-56

Kawachi H, Koike H, Kurihara H, Yaoita E, Orikasa M, Shia MA, Sakai T, Yamamoto T, Salant DJ, Shimizu F. Cloning of rat nephrin: expression in developing glomeruli and in proteinuric states. Kidney Int 2000;57:1949-61

Kestenbaum B, De Boer IH. Urine albumin-to-creatinine ratio: what's in a number? J Am Soc Nephrol 2010;21:1243-4

Kestila M, Lenkkeri U, Mannikko M, Lamerdin J, Mccready P, Putaala H, Ruotsalainen V, Morita T, Nissinen M, Herva R, Kashtan CE, Peltonen L, Holmberg C, Olsen A, Tryggvason $\mathrm{K}$. Positionally cloned gene for a novel glomerular protein-nephrin--is mutated in congenital nephrotic syndrome. Mol Cell 1998;1:575-82

Kim HJ, Cho EH, Yoo JH, Kim PK, Shin JS, Kim MR, Kim CW. Proteome analysis of serum from type 2 diabetics with nephropathy. J Proteome Res 2007a;6:735-43

Kim S, Shin JS, Kim HJ, Fisher RC, Lee MJ, Kim CW. Streptozotocin-induced diabetes can be reversed by hepatic oval cell activation through hepatic transdifferentiation and pancreatic islet regeneration. Lab Invest 2007b;87:702-12

Lee JJ, Yi HY, Yang JW, Shin JS, Kwon JH, Kim CW. Characterization of streptozotocin-induced diabetic rats and pharmacodynamics of insulin formulations. Biosci Biotechnol Biochem 2003;67:2396-401

Liu Y, Shreder KR, Gai W, Corral S, Ferris DK, Rosenblum JS. Wortmannin, a widely used phosphoinositide 3-kinase inhibitor, also potently inhibits mammalian polo-like kinase. Chem Biol 2005;12:99-107

Löwik MM, Groenen PJ, Pronk I, Lilien MR, Goldschmeding R, Dijkman HB, Levtchenko EN, Monnens LA, Van Den Heuvel LP. Focal segmental glomerulosclerosis in a patient homozygous for a CD2AP mutation. Kidney Int 2007;72: 1198-203

Mundel P, Reiser J. Proteinuria: an enzymatic disease of the podocyte? Kidney Int 2010;77:571-80

Neuman RG, Cohen MP. Improved competitive enzymelinked immunoassay (ELISA) for albuminuria. Clin Chim Acta 1989;179:229-37

Okada T, Sakaguchi T, Hatamura I, Saji F, Negi S, Otani H,
Muragaki Y, Kawachi H, Shigematsu T. Tolvaptan, a selective oral vasopressin V2 receptor antagonist, ameliorates podocyte injury in puromycin aminonucleoside nephrotic rats. Clin Exp Nephrol 2009;13:438-46

Pavenstädt $\mathrm{H}$, Kriz W, Kretzler M. Cell biology of the glomerular podocyte. Physiol Rev 2003;83:253-307

Regele HM, Fillipovic E, Langer B, Poczewki H, Kraxberger I, Bittner RE, Kerjaschki D. Glomerular expression of dystroglycans is reduced in minimal change nephrosis but not in focal segmental glomerulosclerosis. J Am Soc Nephrol 2000;11:403-12

Reiser J, Polu KR, Moller CC, Kenlan P, Altintas MM, Wei C, Faul C, Herbert S, Villegas I, Avila-Casado C, Mcgee M, Sugimoto $H$, Brown D, Kalluri R, Mundel P, Smith PL, Clapham DE, Pollak MR. TRPC6 is a glomerular slit diaphragm-associated channel required for normal renal function. Nat Genet 2005;37:739-44

Saleem MA, Ni L, Witherden I, Tryggvason K, Ruotsalainen $\mathrm{V}$, Mundel P, Mathieson PW. Co-localization of nephrin, podocin, and the actin cytoskeleton: evidence for a role in podocyte foot process formation. Am J Pathol 2002;161: 1459-66

Schlondorff J. Nephrin AKTs on actin: The slit diaphragmactin cytoskeleton signaling network expands. Kidney Int 2008;73:524-6

Schmieder S, Nagai M, Orlando RA, Takeda T, Farquhar MG. Podocalyxin activates RhoA and induces actin reorganization through NHERF1 and Ezrin in MDCK cells. J Am Soc Nephrol 2004;15:2289-98

Shibata S, Nagase M, Fujita T. Fluvastatin ameliorates podocyte injury in proteinuric rats via modulation of excessive Rho signaling. J Am Soc Nephrol 2006;17:754-64

Simons M, Hartleben B, Huber TB. Podocyte polarity signalling. Curr Opin Nephrol Hypertens 2009;18:324-30

Smoyer WE, Mundel P. Regulation of podocyte structure during the development of nephrotic syndrome. J Mol Med 1998;76:172-83

Tryggvason K, Wartiovaara J. Molecular basis of glomerular permselectivity. Curr Opin Nephrol Hypertens 2001;10: 543-9

Yousif $\mathrm{MH}$. Phosphoinositide 3-kinase contributes to diabetes-induced abnormal vascular reactivity in rat perfused mesenteric bed. Cell Biochem Funct 2008;26: 451-8

Zheng CX, Chen ZH, Zeng CH, Qin WS, Li LS, Liu ZH. Triptolide protects podocytes from puromycin aminonucleoside induced injury in vivo and in vitro. Kidney Int 2008;74: 596-612

Zhu J, Sun N, Aoudjit L, Li H, Kawachi H, Lemay S, Takano T. Nephrin mediates actin reorganization via phosphoinositide 3-kinase in podocytes. Kidney Int 2008;73:556-66 